\title{
Health Inequality in Health Checkups
}

\author{
Jungun Lee* \\ Department of Family Medicine, Wonkwang University Sanbon Hospital, Wonkwang University School of Medicine, Gunpo, Korea
}

According to the National Health Insurance Corporation's annual report on health checkups in 2016, the percentage of participants undergoing general health checkups increased by $5.1 \%$ from $72.6 \%$ in 2011 to $77.7 \%$ in 2016 ; the national screening program for transitional age increased by $8.6 \%$; and infant health examinations increased by $18.1 \% .{ }^{1)}$ In this situation, many primary care physicians in Korea are participating in national health checkups in their clinic.

In this issue, Shin et al. ${ }^{2)}$ investigated the association between socioeconomic status and adherence to health checkups in a Korean population aged 40 years or older. They categorized health checkups into opportunistic and national health checkups. ${ }^{2)}$ Opportunistic health checkups are screening tests for which participants pay the hospital costs for checkups performed in private healthcare sectors. National health checkups are screening tests including industry-specific health examinations, general health checkups provided by the Korean National Health Insurance Corporation, and health checkups provided by other government organizations. ${ }^{3)}$

They found that individuals of both sexes with a higher socioeconomic status (a high income or more educated) were more likely to undergo health checkups. Socioeconomic status may influence healthcare utilization in various ways. Individuals with a lower socioeconomic status may have difficulty gaining access to the healthcare system due to economic barriers, informational disparity, perceived lack of need, weak support from their employer to use the healthcare system, and fear of additional expenses. ${ }^{4-6)}$

These results were prominent in opportunistic health checkups than in national health checkups. This finding is consistent with previous studies of the characteristics of people who prefer opportunistic health checkups over national health checkups. Hahm et al. ${ }^{7)}$ analyzed the results of the Korean National Health and Nutritional Examination and found that individuals who are at least high school graduates and the upper quartile for income were more likely to undergo opportunistic than national health checkups.

More importantly, however, national health checkups that are conducted across the population also showed this health inequality. The authors explain this result as follows: several distorted stereotypes of national health checkup programs, such as "the 'national' program may be perceived as more untrustworthy than 'opportunistic' programs, or the 'national health checkup program' may be considered as being not for healthy people, but for people with symptoms" might be stronger in low socioeconomic status groups. ${ }^{1)}$ Such misperceptions prevent low socioeconomic status groups from using national health checkups.

Health inequality is an important social, ethical, and health issue worldwide as it is in South Korea. ${ }^{8-10)}$ Encouraging health checkups among individuals with a low socioeconomic status is a good first step to decrease health inequality.

\section{CONFLICT OF INTEREST}

No potential conflict of interest relevant to this article was reported.

\section{REFERENCES}

1. National Health Insurance Service. Annual report on health checkup [Internet]. Wonju: National Health Insurance Service; 2016 [cited 2018 Mar 2]. Available from: https://www. nhis.or.kr/bbs7/boards/B0039/24877.

2. Shin HY, Kang HT, Lee JW, Lim HJ. The association between socioeconomic status and adherence to health check-up in Korean adults, based on the 2010-2012 Korean National Health and Nutrition Examination Survey. Korean J Fam Med 2018;39:114-21. 
3. Cho B, Lee CM. Current situation of national health screening systems in Korea. J Korean Med Assoc 2011;54:666-9.

4. Dunlop S, Coyte PC, McIsaac W. Socio-economic status and the utilisation of physicians' services: results from the Canadian National Population Health Survey. Soc Sci Med 2000;51:123-33.

5. Lee YY, Jun JK, Suh M, Park BY, Kim Y, Choi KS. Barriers to cancer screening among medical aid program recipients in the Republic of Korea: a qualitative study. Asian Pac J Cancer Prev 2014;15:589-94.

6. Lee SH, Joh HK, Kim S, Oh SW, Lee CM, Kwon H. Income disparities in the use of health screening services among university students in Korea: a cross-sectional study of 2479 participants in a university. Medicine (Baltimore) 2016;95:e3681.

7. Hahm MI, Chen HF, Miller T, O'Neill L, Lee HY. Why do some people choose opportunistic rather than organized cancer screening?: the
Korean National Health and Nutrition Examination Survey (KNHANES) 2010-2012. Cancer Res Treat 2017;49:727-38.

8. Kim YM, Kim MH. Health inequalities in Korea: current conditions and implications. J Prev Med Public Health 2007;40:431-8.

9. Closing the gap in a generation: health equity through action on the social determinants of health. Proceedings of an international conference based on the work of the Commission on Social Determinants of Health, 6-7 November 2008, London, UK. Glob Health Promot 2009; Suppl 1:1-118.

10. Mackenbach JP, Bakker MJ; European Network on Interventions and Policies to Reduce Inequalities in Health. Tackling socioeconomic inequalities in health: analysis of European experiences. Lancet 2003;362:1409-14. 